\title{
Conocimiento sobre prevención del covid- 19 en gestantes de la microred de salud del distrito de Yauli, Huancavelica -Perú
}

Mayra Estefany Inga Guillen

2017321012@unh.edu.pe https://orcid.org/0000-0003-2783-0365 Universidad nacional de Huancavelica-Perú.

Geraldine Meza Ramírez

2017321017@unh.edu.pe https://orcid.org/0000-0002-8363-3633 Universidad nacional de Huancavelica-Perú.

Max Junior Paucar Pino

juniormaxpaucarpino@gmail.com https://orcid.org/0000-0002-7660-2169 Universidad nacional de Huancavelica-Perú.

Lina Yubana Cardenas Pineda
https://orcid.org/0000-0001-5715-7031
lina.cardenas@unh.edu.pe
Universidad nacional de Huancavelica-Perú

\section{RESUMEN}

Objetivo: Determinar el conocimiento sobre prevención del COVID-19 en gestantes de Yauli-Huancavelica. Metodología: Investigación descriptivo, observacional, prospectivo, transversal, Población: 185 gestantes de la Microred del distrito de Yauli. Resultados: la mayoría de las gestantes viven en zonas rurales (89.19\%), poseen grado de instrucción secundaria (64.86\%), son amas de casa (72.97\%), más de la mitad son evangélicos (54.05\%), el 62.16\% son adultos jóvenes (20 a 34 años). Los medios por el que se informaron fueron: radio $54.59 \%$, televisión $33.51 \%$, celular $22.70 \%$. La mayoría conoce los medios de transmisión (89.19\%), periodo de incubación (73.51\%) y las medidas de prevención: en cuanto al lavado de manos, el $24.32 \%$ conoce la frecuencia y el $70.81 \%$ el tiempo, hay bajo conocimiento en la frecuencia y lugares en el que debe usarse la mascarilla (5.95\%), el 55.68\% conoce sobre distanciamiento, el $46.49 \%$ sabe cómo actuar frente a los signos y síntomas. Los signos y síntomas más conocidos fueron: 
fiebre, diarrea, sensación de falta de aire, dolor de pecho, perdida del gusto, perdida del olfato, dolor abdominal. Las creencias: tomar infusiones, sangre de cuy y llama, hacer gárgara de sal, orine podrido, ajo con cebolla, El 66.49\% rechaza la vacuna. Conclusión: El nivel de conocimiento es regular a bueno.

Palabras clave: covid-19; gestante, conocimiento; medidas preventivas. 


\title{
Knowledge about covid-19 prevention in pregnant women in the health micronetwork of the district of Yauli, Huancavelica -Peru
}

\begin{abstract}
ABSTRAC
Objective: To determine the knowledge about prevention of COVID-19 in pregnant women from Yauli-Huancavelica. Methodology: Descriptive, observational, prospective, cross-sectional research. Population: 185 pregnant women from the Micronetwork of the Yauli district. Results: most of the pregnant women live in rural areas $(89.19 \%)$, have a secondary education degree $(64.86 \%)$, are housewives $(72.97 \%)$, more than half are evangelical (54.05\%), 62.16\% are young adults (20 to 34 years). The means by which they were informed were: radio $54.59 \%$, television $33.51 \%$, cell phone $22.70 \%$. Most know the means of transmission (89.19\%), incubation period $(73.51 \%)$ and prevention measures: regarding hand washing, $24.32 \%$ know the frequency and $70.81 \%$ the time, there is little knowledge in the frequency and places where the mask should be used (5.95\%), 55.68\% know about distancing, 46.49\% know how to act in the face of signs and symptoms. The best known signs and symptoms were: fever, diarrhea, shortness of breath, chest pain, loss of taste, loss of smell, abdominal pain. Beliefs: drink infusions, guinea pig and llama blood, gargle with salt, rotten urine, garlic with onion, $66.49 \%$ reject the vaccine. Conclusion: The level of knowledge is fair to good
\end{abstract}

Keywords: covid-19; pregnant woman; knowledge; preventive measures.

Artículo recibido: 20 diciembre. 2021 Aceptado para publicación: 10 enero 2022 Correspondencia: 2017321012@unh.edu.pe Conflictos de Interés: Ninguna que declarar 


\section{INTRODUCCIÓN}

El COVID - 19, es un Síndrome Respiratorio Agudo Severo (SARS-CoV-2), responsable de una devastadora pandemia que se reconoció como tal, el 11 de marzo del 2021, causó mucho dolor en todo el mundo y nos dejó colapsado los servicios de salud (Rivero Ramos \& Donado García, 2021). El virus se diseminó por todo el Perú, afectando con mayor crudeza a las Regiones de Lima en primera instancia, seguido de Loreto, Callao y Lambayeque(Placeres Hernández, 2020).

El embarazo trae consigo muchos cambios en el cuerpo de la madre. Entre ellos, la mujer experimenta cambios inmunológicos y estructurales, por lo que están en mayor riesgo de infecciones virales (Espinoza Altamirano \& López Loza, 2021). Existen cambios importantes en la fisiología pulmonar, la cual es más marcada en la segunda mitad del embarazo. El diafragma se eleva aproximadamente $4 \mathrm{~cm}$ debido al crecimiento uterino, mientras que el consumo de oxígeno aumenta en un $20 \%$ y la capacidad residual funcional disminuye de manera significativa, situación que le pone en mayor riesgo a la gestante(Cueva-Buendía et al., 2020).

El COVID - 19, afecta tanto a la gestante y su perinato, está relacionado con abortos, partos pretérminos y distrés respiratorio (Huerta Saenz et al., 2020). En tal situación es necesario fortalecer la prevención, y evaluar el nivel de conocimiento de la prevención del COVID-19.

Estudios sobre conocimiento de prevención del COVID -19, desarrollados por medios virtuales y telefónicos como el de Nepal, se encontraron que la mayoría de las gestantes tienen altos niveles de conocimiento, y algunas creencias como que el virus se transmite por consumir carne de las aves(Singh et al., 2020), asimismo en Huancayo-Perú, se determinó que las gestantes tienen conocimientos adecuados(Pomachagua Valdez, 2020) La vía de transmisión del SARS-COV-2 es de persona a persona por vía respiratoria, con un periodo de incubación de 1 a 14 días, y periodo de incubación media de 5 a 6 días(Maguiña Vargas et al., 2020). Se presenta en la mayoría de casos con un cuadro clínico correspondiente a una infección respiratoria de vías altas, con variedad de sintomatología según grupos de riesgo, presentando una rápida progresión a una neumonía grave y fallo multiorgánico, generalmente fatal en personas de la tercera edad y con presencia de comorbilidades(Abreu et al., 2020).Los síntomas más manifestados son: fiebre, congestión nasal, disnea, malestar, mialgia, perdida del gusto, anosmia, 
agravándose en enfermedades como el síndrome respiratorio agudo severo(Albornoz Toyohama \& Ynfante Izquierdo, 2021). Dentro de los factores de riesgo en donde se presentan mayores complicaciones son: la edad avanzada, inmunodeprimidos gestantes, puérperas y personas con comorbilidades (Huang et al., 2020).Los síntomas Gastrointestinales pueden ser comunes en COVID-19 y en algunos casos ser la primera manifestación incluso antes de fiebre y síntomas respiratorios. Por ello, los clínicos y gastroenterólogos deben estar alertas a estos casos atípicos durante la actual pandemia y sobre la probable transmisión fecal-oral, y considerar medidas preventivas (Schmulson et al., 2020).Como se observa las pocas investigaciones no ha involucrado a las gestantes de las zonas rurales del Perú, por las particularidades en el idioma y los medios de información han tenido poco acceso a información fiable. Generándoles más miedo y temor incluso a asistir a sus atenciones prenatales.

(Durand Llaguento \& Huaman Sinarahua, 2021) realizaron un estudio titulado "Nivel de conocimiento sobre Covid-19 en gestantes atendidas en el Centro De Salud de Corrales, Tumbes agosto-octubre 2021" una investigación descriptiva simple, no experimental, con una población conformada por 192 gestantes, la muestra fue de 98 gestantes, donde obtuvieron los siguientes Resultados: El 70,41\% obtuvieron información a través de TV, el 88,78\% conoce la forma de transmisión del Covid-19. El 69,39\% conoce el periodo de incubación el 77,96 \% conoce las medidas preventivas, el 67,01\% conoce los signos y síntomas de alarma, el 45,91\% recibió atención prenatal de manera presencial y un 96,9\% recibió consejería acerca de Covid-19. Se identificó que el nivel de conocimiento es alto sobre Covid-19 con un 76,26\%.

(Ordoñez et al., 2021) realizaron una investigacion titulada "conocimientos y prácticas de las gestantes sobre la prevención del covid-19” con el Objetivo: Identificar el nivel de conocimientos y las prácticas sobre medidas de prevención de COVID-19 de las gestantes. Metodología: investigación de tipo observacional, descriptiva y trasversal realizada en el Centro de Salud Tipo C "Velasco Ibarra", Machala-Ecuador, con una muestra censal de 100 embarazadas de la consulta de gineco-obstetricia. Resultados: En las gestantes predominó el grado de instrucción bachillerato en 57\%, el 53\% presentaron un nivel de conocimiento adecuado de la transmisión del SARS-CoV-2, el 84\% con un nivel adecuado de conocimiento de las medidas de prevención de COVID-19, el 74\% aplicaban las medidas de prevención, el $83 \%$ utilizan en forma correcta la mascarilla, el 
$60 \%$ aplicaban la secuencia de los pasos de la higiene de las manos y el $55 \%$ el distanciamiento social. En la cual llegaron a la conclusión que a pesar del predominio de un nivel adecuado de conocimiento de las medidas de prevención de COVID-19, con aplicación en forma correcta de la mascarilla, higiene de las manos y distanciamiento social, un importante grupo de embarazadas presentaron conocimientos inadecuados de las medidas de prevención, resaltando el incumplimiento del distanciamiento social, que es la mejor forma para disminuir la propagación del virus.

(Villar Cuba, 2021) realizo un estudio titulado "Conocimiento del COVID 19 en gestantes atendidas en el Centro de Salud de San Vicente de Cañete Año 2021" con el objetivo: Determinar el conocimiento del COVID19 en gestantes atendidas en el Centro de Salud de San Vicente de Cañete año 2021. Metodología: Investigación de tipo básica, no experimental, de corte transversal y de nivel descriptivo. La muestra fue conformada por 122 gestantes, seleccionadas de manera aleatoria, en quienes se aplicó un cuestionario de 14 ítems, se calificó en tres niveles bajo, medio y alto utilizando la técnica de percentil, quedando de la siguiente manera. Resultados: Se observó un predominio de nivel de conocimiento mayormente alto con un $41.8 \%$, como en cada una de sus dimensiones. En torno a los criterios de síntomas con un $72.1 \%$ conocimiento medio; en los signos de gravedad con un $65.6 \%$ en conocimiento alto, en medidas de prevención en un $59.0 \%$ en un nivel de conocimiento medio. Conclusiones: El nivel de conocimiento del COVID 19 es alto en un $41.8 \%$, medio en un $36.1 \%$ y bajo en un $22.1 \%$.

(Paucar Aduviri, 2021) realizo una investigacion titulada "Nivel de conocimiento del COVID-19 en madres gestantes que asisten al Centro de Salud Simón Bolívar, Puno 2021 " tuvo por objetivo determinar el nivel de conocimiento sobre COVID-19 en madres gestantes. La metodología fue de nivel no experimental, con diseño descriptivo y de corte transversal, la muestra de estudio estuvo formada por 97 madres que asisten a sus controles prenatales en dicho establecimiento de salud, para recoger la información se utilizó un cuestionario de 20 interrogantes distribuido en dos dimensiones. Encontrando que el nivel de conocimiento en la dimensión de etiología, medios de transmisión y grupos de riesgo en COVID-19, se encuentra en nivel regular con $41.24 \%$ y bueno $39.18 \%$ en madres gestantes que asisten al Centro de Salud Simón Bolívar. El nivel de conocimiento en la dimensión de cuadro clínico, medidas de prevención y protección por COVID-19, se encuentra en nivel regular con $48.45 \%$ y malo con $47.42 \%$. El nivel de conocimiento 
sobre COVID-19, en madres de primer trimestre es regular con 86.96\%, segundo trimestre con $79.55 \%$ y en tercer trimestre también regular con $80 \%$ en gestantes del Centro de Salud Simón Bolívar. Llegando a la conclusión que el nivel de conocimiento sobre COVID-19 en madres gestantes se encuentra en un nivel regular con el $81.44 \%$. (Alvarez Mateo) realizo una investigacion denominado "Nivel de conocimiento sobre la prevención del Covid-19 en la población que acude al Puesto de Salud de HuananguiSayán 2021" con el objetivo de determinar el Nivel de conocimiento sobre la prevención del Covid-19 en la población que acude al puesto de Salud de Huanangui - Sayán, 2021. El estudio es de tipo básica, en cuanto a su nivel fue descriptivo, respecto del diseño fue no experimental, de corte transversal y tuvo un enfoque cuantitativo. La población estuvo conformada por 66 pobladores que acude al puesto de Salud de Huanangui - Sayán, que fueron a dicho centro de atención entre los meses de marzo a abril de 2021. La técnica utilizada fue la encuesta y el instrumento un cuestionario, el mismo que estuvo conformado por 20 preguntas en 3 dimensiones, con respuestas de tipo Likert. Los resultados llegaron a indicar que la población que acude al puesto de Salud de HuananguiSayán, tiene un bajo nivel de conocimiento sobre de las medidas de prevención, medidas higiénicas y medidas de cuidado que se debe tener ante el Covid19.

(Muñoz-Callol et al., 2020) realizaron una investigacion titulada "Conocimientos sobre la COVID-19 en embarazadas de un hogar materno" con el objetivo: caracterizar el nivel de conocimientos sobre la COVID-19 de las embarazadas internadas en el hogar materno “Casapiedra”, perteneciente al Policlínico Universitario "Guillermo Tejas”, de Las Tunas, de marzo a mayo de 2020. Metodología: se realizó un estudio cuasiexperimental, de intervención educativa, en una muestra de 14 embarazadas internadas en la institución y durante el tiempo declarados en el objetivo. Se aplicó un cuestionario antes y después de la intervención, para determinar el nivel de conocimientos y preocupaciones sobre la COVID-19 y el proceso de parto y postparto. Se evaluaron, además: edades materna y gestacional, nivel de enseñanza educacional terminada y motivo de ingreso. Resultados: antes de intervenir la mayoría de las embarazadas presentaron necesidades de aprendizaje sobre los síntomas, medidas generales y específicas ante la sospecha o confirmación de una gestante portadora de la COVID-19, en la totalidad de los casos manifestaron preocupación sobre la transmisión a su bebé a través de la lactancia materna. Después de intervenir se incrementó el nivel de conocimientos, pero el 71,4 \% manifestó 
preocupación sobre el riesgo de contraer la infección fuera del hogar materno, relacionado con la existencia de enfermedades de base y el propio embarazo. Conclusiones: la intervención educativa fue efectiva, aunque se evidenció la permanente preocupación de las embarazadas por el proceso de parto y postparto en estos tiempos de pandemia.

Por la situación planteada, se investigó el conocimiento acerca de la prevención del COVID-19, en gestantes de las comunidades donde habitan la etnia denominada Chopcca, que tienen como idioma principal al idioma quechua, población aproximada 34 324 habitantes, de los cuales 13923 son hombres y el 14204 mujeres, respecto a la educación 10047 saben leer, 3224 son iletrados y un 24,3\% son analfabetos, por otro lado respecto a la actividad económica, casi el $28 \%$ de la población se dedica a la agricultura, seguido de la actividad del comercio $20 \%$ y en menor porcentajes $4 \%$ en la minería, y actividad artesanal(Kobayashi Gamboa, 2020).Los resultados de la investigación sirven para plantear estrategias pertinentes para fomentar medidas preventivas.

\section{MATERIALES Y METODO}

El presente estudio fue de tipo descriptivo, observacional, prospectivo, transversal(Hernández-Sampieri \& Torres, 2018). Que nos ayudó a identificar el nivel de conocimiento sobre el COVID-19 en específico de las medidas preventivas.

Población: La población con la que se realizó la investigación estuvo constituida por todas las gestantes atendidas en la Microred de Salud del Distrito de Yauli-Huancavelica, Perú constituido por 198 gestantes.

Muestra: La muestra del estudio fue Censal, sin embargo, tuvimos cuatro gestantes que no se les ubicó, y nueve gestantes que no aceptaron participar, en tal sentido la muestra quedó en 185.

\section{TÉCNICAS E INSTRUMENTOS DE RECOLECCIÓN DE DATOS}

Técnica: La encuesta, estuvo orientada a medir el nivel de conocimiento por medio de un cuestionario que se aplicó, a través de entrevista directa con la gestante. previo consentimiento informado.

Instrumento: Cuestionario con 5 (ítems), denominado "Cuestionario del conocimiento sobre prevención del COVID-19", el instrumento fue validado por profesionales entendidos en la materia. Los datos recopilados fueron ingresados a una base de datos de 
IBM SPSS statistics versión 25, paquete estadístico que posibilita el análisis del conocimiento sobre prevención del COVID-19 en gestantes, para el estudio.

Técnicas y procesamiento de análisis de datos estadísticas descriptivas

Para el análisis de los datos recolectados, se usó la estadística descriptiva, que nos permite presentar los resultados en tablas con frecuencias absolutas y porcentuales, Para hallar la frecuencia de esta variable se usó el programa IBM SPSS versión 25.

\section{RESULTADOS}

\section{Presentación e interpretación de datos}

Tabla 01: Características sociodemográficas la población de gestantes de la microred de salud del distrito de Yauli-Huancavelica, en el periodo noviembre 2020 a mayo 2021

\begin{tabular}{lcc}
\hline & Características sociodemográficas & Gestante \\
\cline { 2 - 3 } & $\mathbf{N}=\mathbf{1 8 5}$ & $\%$ \\
\hline Procedencia & & \\
\hline Rural & 165 & $89.19 \%$ \\
\hline Urbano & 20 & $10.81 \%$ \\
\hline Grado de instrucción & \\
\hline Secundaria & 120 & $64.86 \%$ \\
\hline Primaria & 53 & $28.65 \%$ \\
\hline Ocupación actual & & \\
\hline Su casa & 135 & $72.97 \%$ \\
\hline Agricultura y pastoreo & 42 & $22.70 \%$ \\
\hline Religión & & \\
\hline Evangélicos & 100 & $54.05 \%$ \\
\hline Católico & 84 & $45.41 \%$ \\
\hline Edad & & \\
\hline $20-34$ & 115 & $62.16 \%$ \\
\hline $15-19$ & 44 & $23.78 \%$ \\
\hline $35-44$ & 26 & $14.05 \%$ \\
\hline
\end{tabular}

Fuente: Instrumento de recolección de datos "Nivel de conocimiento sobre prevención del COVID-19 en gestantes de la Microred de Salud del distrito de Yauli-Huancavelica, en el periodo noviembre 2020 a mayo 2021".

Interpretacion: En la tabla 01: Del 100\% de gestantes el 89.19\% habitan en la parte rural y el $10.81 \%$ procede de la parte urbana, en el grado de instrucción tenemos secundaria el $64.86 \%$, seguido de primaria en un $28.65 \%$, iletrada en un $3.24 \%$ y superior técnico e universitario el $1.62 \%$ respectivamente, el $72.97 \%$ se dedican a su casa y el $22.70 \%$ se 
dedica a la agricultura y el pastoreo, el 3.78\% trabajo independiente seguido del $0.54 \%$ que tiene trabajo del estado, respecto a la religión son evangélicas el 54.05\%, católica el $45.41 \%$, y otras religiones el $0.54 \%$, la edad predominante fue de 20 a 34 años con un $62.16 \%$, adolescentes de 15 a 19 años el $23.78 \%$, y el $14.05 \%$ tienen entre 35 a 44 años.

Tabla 02: Medios de comunicación por el cual se informaron las gestantes de la microred de salud del distrito de yauli-huancavelica, en el periodo noviembre 2020 a mayo 2021. Respecto a las medidas preventivas para evitar el contagio del covid-19.

\begin{tabular}{l|cc}
\hline \multicolumn{3}{c}{ GESTANTE } \\
\hline Medios por el que se informaron del covid-19 & SE INFORMA \\
\hline \multirow{3}{*}{ Radio } & N=85 & \% \\
\cline { 2 - 3 } Televisión & 101 & $54.59 \%$ \\
Celular & 62 & $33.51 \%$ \\
Personal de salud & 42 & $22.70 \%$ \\
Familiares o amigos & 9 & $4.86 \%$ \\
& 3 & $1.62 \%$
\end{tabular}

Fuente: Instrumento de recolección de datos "Nivel de conocimiento sobre prevención del COVID-19 en gestantes de la Microred de Salud del distrito de Yauli-Huancavelica, en el periodo noviembre 2020 a mayo 2021 ",

Interpretacion: En la Tabla 02: Del total de gestantes, el 54.59\% (101) reconocen que se informaron por radio y el 33.51\% (62) por televisión y el $22.70 \%$ (42) por celular y un $4.86 \%$ (9) fue informada por el personal de salud y un $1.62 \%$ (3) por familiares o amigos.

Tabla 03: Conocimiento general de las gestantes sobre el covid-19 en la microred de salud del distrito de Yauli-Huancavelica, en el periodo noviembre 2020 a mayo 2021.

\begin{tabular}{ll|rr|rrr}
\hline \multicolumn{2}{l|}{ Conocimientos generales sobre covid-19 } & \multicolumn{2}{c|}{ Conoce } & \multicolumn{2}{c}{ No conoce } \\
\hline \multicolumn{2}{l|}{$\begin{array}{l}\text { ¿Reconoce al covid-19 como una } \\
\text { enfermedad? }\end{array}$} & 166 & $89.73 \%$ & 19 & $10.27 \%$ \\
\hline Conoce medios de transmisión & 165 & $89.19 \%$ & 20 & $10.81 \%$ \\
\hline Conoce periodo de incubación & 136 & $73.51 \%$ & 49 & $26.49 \%$ \\
\hline
\end{tabular}


Fuente: Instrumento de recolección de datos "Nivel de conocimiento sobre prevención del COVID-19 en gestantes de la Microred de Salud del distrito de Yauli-Huancavelica, en el periodo noviembre 2020 a mayo 2021".

Interpretacion: En la tabla 03: del 100\% de las gestantes el 89.73\% (166) reconoce al COVID-19 como enfermedad y el 10.27\% (19) no reconoce a la COVID -19 cómo una enfermedad, el 89.19\% (165) conoce los medios de transmisión y el 10.81\% (20) no conoce los medios como se transmite, el $73.51 \%$ (136) conoce el periodo de incubación y el 26.49\% (49) desconoce el periodo de incubación.

Tabla 04: Conocimiento sobre prevención del covid-19 por las gestantes en la microred de salud distrito de yauli-huancavelica, en el periodo noviembre 2020 a mayo 2021

\begin{tabular}{l|ccc|c}
\hline \multirow{2}{*}{ Conocimiento sobre prevención } & \multicolumn{5}{c}{ Conoce } & \multicolumn{2}{c}{ No conoce } \\
\cline { 2 - 5 } & N=185 & \% & n=185 & $\%$ \\
\hline $\begin{array}{l}\text { Conoce la frecuencia con la que te } \\
\text { lavas las manos después de haber } \\
\text { estado en un lugar público. }\end{array}$ & 45 & $24.32 \%$ & 140 & $75.68 \%$ \\
\hline $\begin{array}{l}\text { Conoce el tiempo de lavado de manos } \\
\text { Sabe la frecuencia con la que debe }\end{array}$ & 131 & $70.81 \%$ & 54 & $29.19 \%$ \\
\hline $\begin{array}{l}\text { usar mascarilla en lugares públicos } \\
\text { Conoce el uso adecuado de la } \\
\text { mascarilla }\end{array}$ & 184 & $59.95 \%$ & 174 & $94.05 \%$ \\
\hline $\begin{array}{l}\text { Conoce a que distancia debe de estar } \\
\text { de otra persona }\end{array}$ & 103 & $55.68 \%$ & 82 & $44.32 \%$ \\
\hline $\begin{array}{l}\text { Sabe que debe hacer si tiene dos más } \\
\text { signos y síntomas }\end{array}$ & 86 & $46.49 \%$ & 99 & $53.51 \%$ \\
\hline
\end{tabular}

Fuente: Instrumento de recolección de datos "Nivel de conocimiento sobre prevención del COVID-19 en gestantes de la Microred de Salud del distrito de Yauli-Huancavelica, en el periodo noviembre 2020 a mayo 2021"."

Interpretacion: En la tabla 04: Sobre las medidas preventivas, de las 185 gestantes, el 99.46\% (184) conoce el uso adecuado de la mascarilla, y el $0.54 \%$ (1) no conoce el uso adecuado de la mascarilla, el 70.81\% (131) conoce el tiempo de lavado de manos, el $29.19 \%$ (54) no conoce el tiempo de lavado de manos, el 55.68\% (103) conoce la distancia que debe de guardar frente a otra persona, seguido del $44.32 \%$ (82) que no conoce a que distancia debe de estar de una persona, el 24.32\% (45) sabe la frecuencia con que debe de lavarse las manos después de haber estado en un lugar público, y el 75.68\%(140) que no conoce la frecuencia con la que se debe lavar las manos después de estar en lugares 
públicos, el 46.49\% (86) sabe qué hacer si presenta dos signos y síntomas, seguido del $53.51 \%$ que no sabe qué hacer si tiene dos o más síntomas.

Tabla 05: Conocimiento de los signos y síntomas de covid-19 por las gestantes de la microred de salud del distrito de yauli-huancavelica, en el periodo noviembre 2020 a mayo 2021

\begin{tabular}{|c|c|c|}
\hline \multirow[t]{2}{*}{ SIGNOS Y SÍNTOMAS } & \multicolumn{2}{|c|}{ CONOCE } \\
\hline & $\mathrm{N}=185$ & $\%$ \\
\hline FIEBRE MAYOR A $38^{\circ} \mathrm{c}$ POR MÁS DE DOS DÍAS. & 176 & $95.14 \%$ \\
\hline Diarrea & 118 & $63.78 \%$ \\
\hline Sensación de falta de aire. & 95 & $51.35 \%$ \\
\hline Dolor de pecho. & 80 & $43.24 \%$ \\
\hline Pérdida del gusto & 80 & $43.24 \%$ \\
\hline Pérdida del olfato & 52 & $28.11 \%$ \\
\hline Dolor abdominal & 35 & $18.92 \%$ \\
\hline Desorientación o confusión & 8 & $4.32 \%$ \\
\hline No conoce ningún signo y síntoma & 6 & $3.24 \%$ \\
\hline
\end{tabular}

Fuente: Instrumento de recolección de datos "Nivel de conocimiento sobre prevención del COVID-19 en gestantes de la Microred de Salud del distrito de Yauli-Huancavelica, en el periodo noviembre 2020 a mayo 2021 "

Interpretacion: En la tabla 05: Al indagar sobre el conocimiento de los signos y síntomas del COVID -19, se encuentra que casi todas $95.14 \%$ (176) reconocen como un signo a la fiebre, a la diarrea en un $63.78 \%$ (118), la sensación de falta de aire el 51.35\% (95), dolor de pecho un $43.24 \%$ (80), perdida del gusto el $43.24 \%$ (80) y perdida del olfato $28.11 \%$ (52), dolor abdominal el 18.92\% (35).

Tabla 06: Conocimiento que tuvieron respecto a las prácticas caseras y creencias para la prevención del covid-19 de las gestantes de la microred de salud del distrito de YauliHuancavelica, en el periodo noviembre 2020 a mayo 2021

\begin{tabular}{|l|c|c|}
\hline \multicolumn{1}{c}{ Mitos y Creencias } & \multicolumn{2}{c}{ GESTANTES } \\
\hline & $\mathbf{N = 1 8 5}$ & $\%$ \\
\cline { 2 - 3 } Hierbas & 152 & $82.16 \%$ \\
Usted hace gárgaras de sal & 88 & $47.57 \%$
\end{tabular}




\begin{tabular}{l|cc} 
Gárgara de orine podrido con sal negra & 82 & $44.32 \%$ \\
Gárgaras de ajos y cebolla & 74 & $40.00 \%$ \\
Toma sangre de cuy & 64 & $34.59 \%$ \\
Toma sangre de llama & 24 & $12.97 \%$ \\
Otros & 8 & $4.32 \%$
\end{tabular}

Fuente: Instrumento de recolección de datos "Nivel de conocimiento sobre prevención del COVID-19 en gestantes de la Microred de Salud del distrito de Yauli-Huancavelica, en el periodo noviembre 2020 a mayo 2021".

Interpretacion: En la tabla 06: Como prácticas caseras de prevención del COVID -19, de las 185 gestantes, el 82.16\% (152) consume diversas hierbas en infusión, 47.57\% (88) hace gárgara de agua con sal, el $44.32 \%$ (82) hace gárgara de orine podrido y sal negra, el 40\% (74) hace gárgara de ajos y cebolla, el 34.59\% (64) toma sangre de cuy, el 12.97\% (24) toma sangre de llama seguido del $4.32 \%$ que tiene otra práctica de prevención frente al COVID-19.

Tabla 07: Percepción que tuvieron respecto a la vacuna del covid-19 las gestantes de la microred de salud del distrito de yauli-huancavelica, en el periodo noviembre 2020 a mayo 2021

\begin{tabular}{lcccc}
\hline \multicolumn{1}{c}{$\begin{array}{c}\text { Actitud frente a la } \\
\text { vacuna }\end{array}$} & SI & N & NO & \% \\
\hline $\begin{array}{l}\text { Usted se vacunaría para } \\
\text { prevenir la enfermedad de } \\
\text { covid-19 }\end{array}$ & 62 & $33.51 \%$ & 123 & $66.49 \%$ \\
\hline
\end{tabular}

Fuente: instrumento de recolección de datos "Nivel de conocimiento sobre prevención del COVID-19 en gestantes de la Microred de Salud del distrito de Yauli-Huancavelica, en el periodo noviembre 2020 a mayo 2021".

Interpretación: En la tabla 07: Se observa la percepción frente a la vacuna del COVID19 que del 100\% de gestantes solo el 33.51\% (62) se vacunaría contra el COVID -19, y el $66.49 \%$ (123) no se vacunaría.

\section{DISCUSIÓN DE RESULTADOS.}

El presente estudio se realizó en el distrito de Yauli, Huancavelica-Perú en gestantes. La etnia Chopcca es una de las pocas que habitan en la sierra del Perú, población que tienen su propia cosmovisión de la salud y la enfermedad, creyente de curar las enfermedades a 
base de productos naturales, es por ello que para el COVID-19 suelen usar ciertos remedios caseros que acuñan sus propiedades preventivas y curativas.

En su mayoría habitan en la zona rural, tienen grado de instrucción secundaria, la mayoría se dedica a su casa y la cuarta parte a la agricultura y pastoreo, un poco más de la mitad de las gestantes tienen religión evangélica 54.05\%; la edad predominante fue de 20 a 34 años $62.16 \%$, lo cual coincide en algunos aspectos con la investigación realizada en Huancayo, (Pomachagua Valdez, 2020). El perfil sociodemográfico identificadas constituyen barreras para una buena información, con respecto a la prevención del COVID-19. Lo cual nos invita a adecuar nuestro sistema de información y educación para la salud según el contexto sociodemográfico y además debemos elegir con los medios por el que se va transmitir, considerando que la mayoría se informan por radio y televisión, y solo la cuarta parte a través de los celulares, esto se bebe a la conectividad y analfabetismo tecnológico que tenemos en especial en las zonas rurales, en este contexto es importante que el personal de salud de la jurisdicción sea protagonista de los procesos de educación con pertinencia cultural.

Respecto al conocimiento general en cuanto al COVID-19, en su mayoría las gestantes conocen acerca del COVID-19, resultados semejantes al encontrado en Cañete (Villar Cuba, 2021). El conocimiento se adquirió por la ansiedad y estrés vivido de forma obligatoria. Sin embargo cuando se evalúa las medidas de prevención si encontramos diferencias, en el presente estudio el $99.46 \%$ conoce el uso adecuado de la mascarilla, el $70.81 \%$ el tiempo de lavado de manos, el 55.68\% la distancia que debe de guardar frente a otra persona, a diferencia del estudio de cañete, que identifica que el 66.5\% no conocía acerca de los contactos cercanos, los puntos débiles de prevención de COVID - 19 , se encuentra en la frecuencia de lavado de manos, y la frecuencia y los momentos del uso de la mascarilla, menos de la mitad (46.49\%) sabe qué hacer si presenta dos signos para no contagiar a los demás, donde, es importante tener en cuenta las recomendaciones dadas en un artículo de revisión de manejo práctico de gestantes con COVID - 19.

La mayoría conoce, los signos y síntomas del COVID -19, como la fiebre, diarrea, sensación de falta de aire, dolor de pecho, entre otros, semejante a lo encontrado por (Muñoz-Callol et al., 2020) donde el 71,4\% conocía los síntomas principales del COVID19. 
En cuanto al conocimiento respecto a las prácticas caseras de prevención del COVID 19, de las 185 gestantes, el $82.16 \%$, de las gestantes reportaron conocer y haber practicado el consumo de diversas hierbas en infusión, hacer gárgara de sal, orine podrido y sal negra, ajos y cebolla, tomar sangre de cuy y llama, con la creencia que alguno de ellos es preventivo y otros como la sangre curativos, que se le dan cuando tenga signos y síntomas. Frente a la percepción que tuvieron respecto a las vacunas, del total de gestantes solo el $33.51 \%$ se vacunaría contra el COVID -19, y el 66.49\% no se vacunaría, ya que de cierto modo temen a que se pueda presentar más adelante alguna reacción adversa y deteriorar su salud. Este hallazgo nos manifiesta que debemos trabajar más en información y educación con respecto a las vacunas contra el COVID - 19, haciendo incidencia en los líderes comunales.

\section{CONCLUSIÓN}

El nivel de conocimiento sobre prevención del COVID - 19 de las gestantes, es regular a bueno, sin embargo, existen debilidades en cuanto al lavado de manos y uso de mascarilla, además es necesario realizar una intervención de educación para la salud, con adecuación intercultural a través de los profesionales de la salud, con participación social, para aclarar las creencias y mitos sobre el COVID-19, y sensibilizar en la importancia de la vacuna.

\section{REFERENCIAS BIBLIOGRAFICAS}

Abreu, M. R. P., Tejeda, J. J. G., \& Guach, R. A. D. (2020). Características clínicoepidemiológicas de la COVID-19. Revista Habanera de Ciencias Médicas, 19(2), $1-15$.

Albornoz Toyohama, R. H., \& Ynfante Izquierdo, E. A. (2021). Características clínicas, laboratoriales del embarazo y resultados perinatales en gestantes con infección de SARS-Cov-2 en una clínica privada.

Alvarez Mateo, E. L. Nivel de conocimiento sobre la prevención del Covid-19 en la población que acude al Puesto de Salud de Huanangui-Sayán 2021.

Cueva-Buendía, L. A., Jesús-Balbín, E. J., \& Buendía-Suazo, J. J. (2020). Comentarios acerca de la salud pública y el cuidado materno fetal y neonatal en la pandemia por ele virus SARS-CoV-2. Revista Internacional de Salud Materno Fetal, 5(2), c4-6.

Durand Llaguento, C. d. M., \& Huaman Sinarahua, A. d. P. (2021). Nivel de conocimiento sobre Covid-19 en gestantes atendidas en el Centro De Salud de Corrales, 
Tumbes agosto-octubre 2021 Universidad Privada de Huancayo Franklin Roosevelt].

Espinoza Altamirano, M. F., \& López Loza, Á. E. (2021). COMPLICACIONES PERINATALES ASOCIADAS AL EMBARAZO DE MADRES ADOLESCENTES

Hernández-Sampieri, R., \& Torres, C. P. M. (2018). Metodología de la investigación (Vol. 4). McGraw-Hill Interamericana México^ eD. F DF.

Huang, C., Wang, Y., Li, X., Ren, L., Zhao, J., Hu, Y., Zhang, L., Fan, G., Xu, J., \& Gu, X. (2020). Clinical features of patients infected with 2019 novel coronavirus in Wuhan, China. The lancet, 395(10223), 497-506.

Huerta Saenz, I. H., Elías Estrada, J. C., Campos Del Castillo, K., Muñoz Taya, R., \& Coronado, J. C. (2020). Características materno perinatales de gestantes COVID19 en un hospital nacional de Lima, Perú. Revista Peruana de Ginecología y Obstetricia, 66(2).

Kobayashi Gamboa, E. L. (2020). Acceso a los servicios de salud de gestantes adolescentes: Análisis socioeconómicos utilizando la encuesta ENDES-INEI, Perú 2014-2018.

Maguiña Vargas, C., Gastelo Acosta, R., \& Tequen Bernilla, A. (2020). El nuevo Coronavirus y la pandemia del Covid-19. Revista Medica Herediana, 31(2), 125131.

Muñoz-Callol, J. L., Ibert-Muñoz, C., Jiménez-Martínez, D., Chacón-Ávila, J. R., \& Torres-Herrera, S. M. (2020). Conocimientos sobre la COVID-19 en embarazadas de un hogar materno. Revista Electrónica Dr. Zoilo E. Marinello Vidaurreta, 45(6).

Ordoñez, M. A. J., Segovia, C. B. V., \& Salinas, S. M. S. (2021). CONOCIMIENTOS Y PRÁCTICAS DE LAS GESTANTES SOBRE LA PREVENCIÓN DEL COVID19. Enfermería Investiga, 6(5), 24-30.

Paucar Aduviri, Y. O. (2021). Nivel de conocimiento del covid-19 en madres gestantes que asisten al Centro de Salud Simón Bolívar, Puno 2021.

Placeres Hernández, J. F. (2020). Pesquisa activa, contribución desde la Atención Primaria de Salud para el control de la COVID-19. Revista Médica Electrónica, 42(4), 2148-2159. 
Pomachagua Valdez, E. V. (2020). CONOCIMIENTO DE LAS MEDIDAS PREVENTIVAS FRENTE A LA PANDEMIA COVID-19, POR LAS GESTANTES CS CHILCA-HUANCAYO, 2020.

Rivero Ramos, Y. d. C., \& Donado García, M. D. (2021). Incidencia de la pandemia del covid-19 en el estrés laboral de los trabajadores de la IPS salud Tierralta SAS.

Schmulson, M., Dávalos, M., \& Berumen, J. (2020). Beware: Gastrointestinal symptoms can be a manifestation of COVID-19. Revista de Gastroenterología de México (English Edition), 85(3), 282-287.

Singh, V., Singh, S., Biswal, A., Kesarkar, A. P., Mor, S., \& Ravindra, K. (2020). Diurnal and temporal changes in air pollution during COVID-19 strict lockdown over different regions of India. Environmental Pollution, 266, 115368.

Villar Cuba, R. A. (2021). Conocimiento del covid 19 en gestantes atendidas en el Centro de Salud de San Vicente de Cañete Año 2021. 